\title{
P-0624 - Clinico-radiological characteristics and correlates of lipohypertrophy in insulin injecting diabetes mellitus patients
}

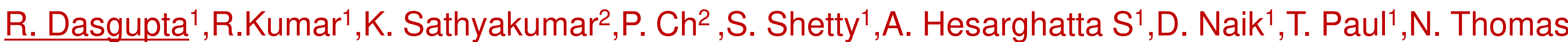

${ }^{1}$ Department of Endocrinology, ${ }^{2}$ Department of Radiology, Christian Medical College Vellore, India.

\section{AIMS AND OBJECTIVES :}

口To study the prevalence and characteristics of Lipohypertrophy (LH) in patients with diabetes mellitus detected by a) clinical examination and b) Ultrasonography

पTo study the risk factors associated with clinically detected Lipohypertrophy in these patients with Diabetes Mellitus

口To study the impact of clinically detected Lipohypertrophy on glycemic control in these patients with diabetes mellitus

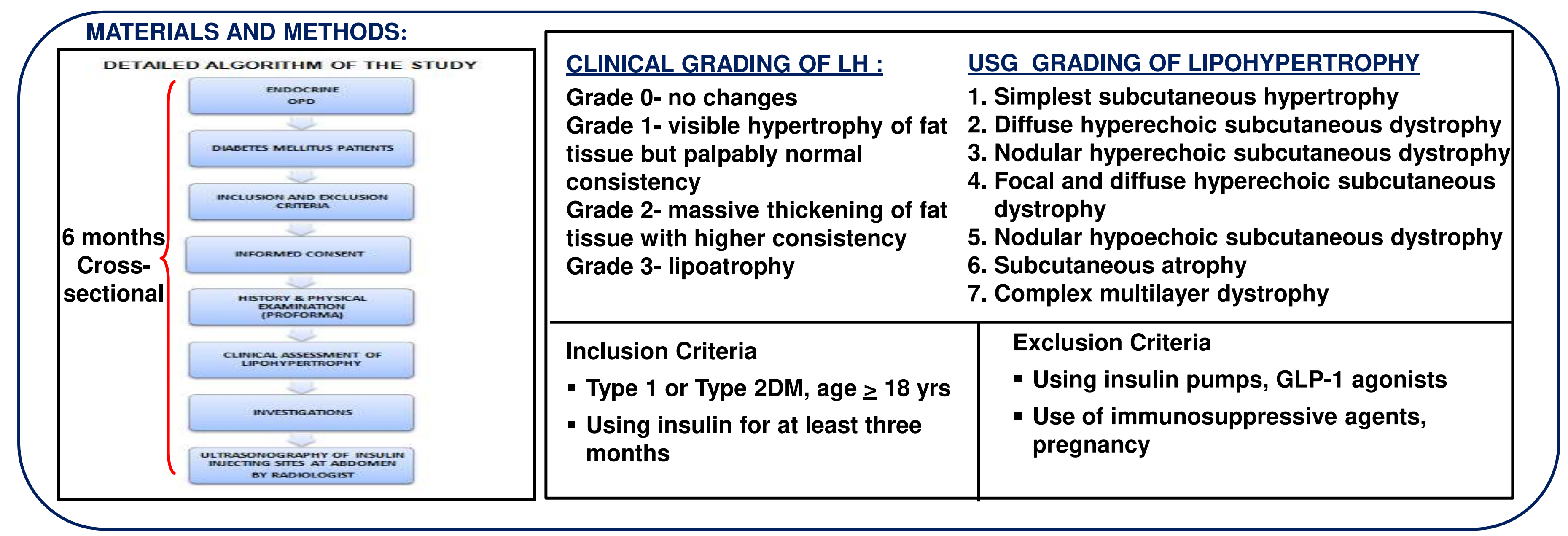

RESULTS AND ANALYSIS :

\begin{tabular}{|c|c|c|c|c|}
\hline \multicolumn{2}{|c|}{ Baseline Characteristics } & Type $1(\mathrm{~N}=15)$ & \multicolumn{2}{|c|}{ Type $2(\mathrm{~N}=73)$} \\
\hline \multicolumn{2}{|l|}{ Age (years) } & $25.20 \pm 7.28$ & \multicolumn{2}{|c|}{$56.74 \pm 10.35$} \\
\hline \multicolumn{2}{|l|}{ BMI $\left(\mathrm{kg} / \mathrm{m}^{2}\right)$} & $21.24 \pm 4.36$ & \multicolumn{2}{|c|}{$27.51 \pm 4.66$} \\
\hline \multicolumn{2}{|c|}{ Duration of DM (years) } & $16.27 \pm 5.88$ & \multicolumn{2}{|c|}{$42.95 \pm 9.95$} \\
\hline \multicolumn{2}{|c|}{ Insulin use(months) } & $100.67 \pm 84.04$ & \multicolumn{2}{|c|}{$73.75 \pm 78.65$} \\
\hline \multicolumn{2}{|c|}{ Total insulin dose (IU/day) } & $44.40 \pm 16.52$ & \multicolumn{2}{|c|}{$57.58 \pm 40.80$} \\
\hline \multicolumn{2}{|l|}{ HbA1c (\%) } & $7.96 \pm 3.04$ & \multicolumn{2}{|c|}{$9.08 \pm 1.85$} \\
\hline \multicolumn{2}{|c|}{ Variable } & $\begin{array}{c}\text { Clinically LH } \\
\text { present }(n=60)\end{array}$ & $\begin{array}{l}\text { Clinically LH } \\
\text { absent }(n=28)\end{array}$ & p-value \\
\hline \multirow[t]{2}{*}{ Insulin use } & $<5$ years & $29(48.3 \%)$ & 20 (71.4\%) & \multirow[t]{2}{*}{0.001} \\
\hline & $>5$ years & $31(51.7 \%)$ & $8(28.6 \%)$ & \\
\hline \multirow[t]{2}{*}{ Needle re-use } & $<3$ times & $22(36.7 \%)$ & $16(59.3 \%)$ & \multirow[t]{2}{*}{0.001} \\
\hline & $>3$ times & $38(63.3 \%)$ & $11(40.7 \%)$ & \\
\hline \multirow[t]{2}{*}{ Site rotation } & Yes & $58(96.7 \%)$ & $19(67.9 \%)$ & \multirow[t]{2}{*}{0.001} \\
\hline & No & $2(3.3 \%)$ & $9(32.1 \%)$ & \\
\hline \multirow[t]{2}{*}{ Needle length } & $4 \mathrm{~mm}$ & $7(11.7 \%)$ & $5(17.9 \%)$ & \multirow[t]{2}{*}{0.54} \\
\hline & $6 \mathrm{~mm}$ & $53(88.3 \%)$ & $23(82.1 \%)$ & \\
\hline \multirow[t]{2}{*}{ Hypoglycemia } & Present & $24(40 \%)$ & $10(35 \%)$ & \multirow[t]{2}{*}{0.02} \\
\hline & Absent & $36(60 \%)$ & $18(65 \%)$ & \\
\hline \multicolumn{2}{|c|}{ Hba1c $>8 \%$} & $25(42 \%)$ & $9(33 \%)$ & 0.01 \\
\hline \multicolumn{2}{|c|}{ Insulin/day dose } & $61.6 \pm 23.3 \mathrm{U}$ & $41.9 \pm 18.3 \mathrm{U}$ & 0.002 \\
\hline
\end{tabular}

Factors associated with increased risk of Lipohypertrophy Univariate regression:

$\square$ Site change frequency ( OR 13.7, $\mathrm{Cl} 2.72-69.23, \mathrm{p}=0.001$ )

aNeedle change frequency ( OR $2.5, \mathrm{Cl} 0.99-6.36, \mathrm{p}=0.04$ )

DLevel of education (OR 2.84, Cl 1.09-7.39, $\mathrm{p}=0.03$ )

Multivariate regression: Site change(OR $-0.35, \mathrm{Cl}-0.78-0.19, \mathrm{p}=0.002)$
Lipohypertrophy: Clinical Grading

DGrade 0 DGrade 1 DGrade 2 DGrade 3

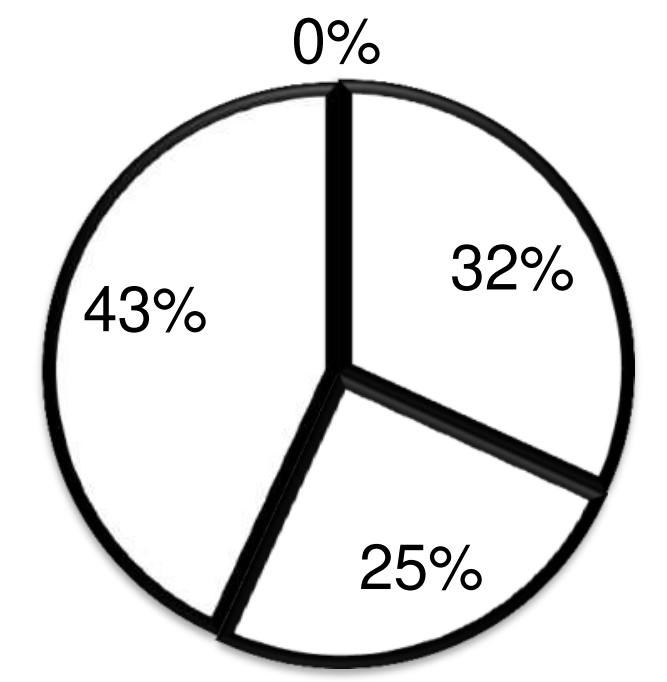

Lipohypertrophy: USG grading

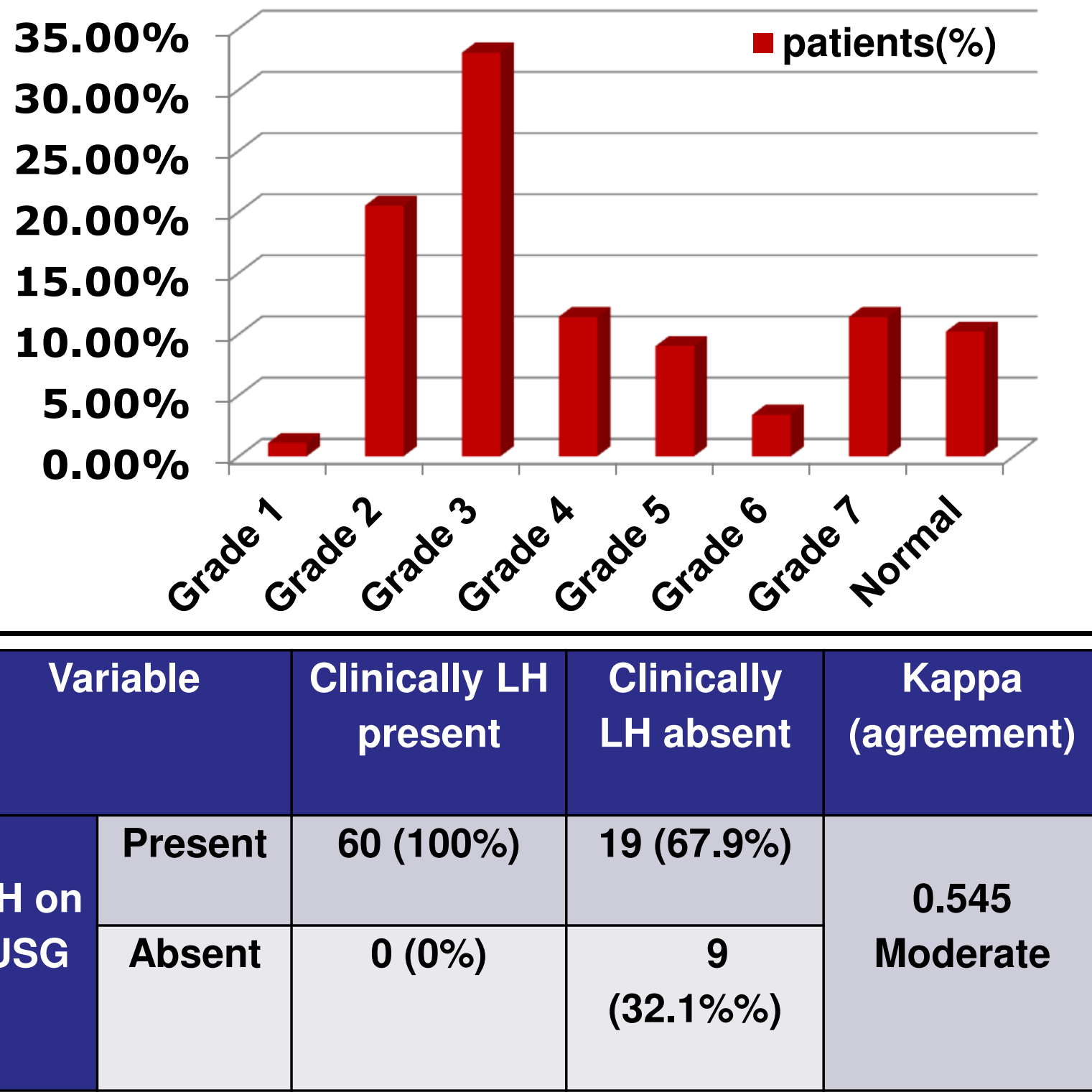

The prevalence of lipohypertrophy by clinical assessment was $68.2 \%$ and by USG assessment was $89.8 \%$ in our study

- Grade 2 commonest clinical LH type, Nodular and diffuse hyperechoic commonest USG type of LH

USG useful adjunct to clinical assessment of lipohypertrophy 